\title{
EFECTO DEL Azotobacter sp. Y EL Azospirillum sp. EN EL RENDIMIENTO DE ALGODÓN DE COLOR (Gossypium barbadense L.) EN EL FUNDO LOS PICHONES, TACNA 2015
}

\author{
EFFECT OF Azotobacter sp. AND Azospirillum sp. IN THE YIELD OF COLOR COTTON (Gossypium \\ barbadense $\mathrm{L}_{5}$.) IN THE FUND PICHONES, TACNA 2015
}

${ }^{1}$ Nelly Arévalo Solsol, ${ }^{2}$ Rosario Zegarra Zegarra

\begin{abstract}
RESUMEN
El objetivo de la presente investigación fue comparar los efectos del Azotobacter sp. y el Azospirillum sp. en el rendimiento del algodón de color (Gossypium barbadense L.) en el fundo Los Pichones- Tacna, paya cuyo efecto, se empleó el diseño experimental de DBCA con 5 tratamientos y 4 Bloques. Los tratamientos fueron: $2 \mathrm{~kg} / \mathrm{m}^{2} \mathrm{M} . \mathrm{O}+$ 60-100-120 NPK ( $\left(\mathrm{T}_{1}\right), 2 \mathrm{~kg} / \mathrm{m}^{2}$ M.O+ Azospirillum sp. $\left(\mathrm{T}_{2}\right), 2 \mathrm{~kg} / \mathrm{m}^{2} \mathrm{M} . \mathrm{O}+$ Azotobacter sp. $\left(\mathrm{T}_{3}\right), 2 \mathrm{~kg} / \mathrm{m}^{2} \mathrm{M} . \mathrm{O}+$ Azospirillum sp. + Azotobacter sp. $\left(\mathrm{T}_{4}\right), 120-100-120$ NPK $\left(\mathrm{T}_{5}\right)$. Los resultados indican que los rendimientos con aplicación de materia orgánica, Azospirillum sp. y Azotobacter $\mathrm{sp}\left(\mathrm{T}_{4}\right)$ son superiores a los tratamientos $2 \mathrm{~kg} / \mathrm{m}^{2} \mathrm{M} . \mathrm{O}+$ Azospirillum sp $\left(\mathrm{T}_{2}\right)$ y $2 \mathrm{~kg} / \mathrm{m}^{2} \mathrm{M} . \mathrm{O}+$ Azotobacter $\mathrm{sp}$. $\left(\mathrm{T}_{3}\right)$, siendo estos tratamientos superados por los tratamientos químicos $2 \mathrm{~kg} / \mathrm{m}^{2}$ M.O + 60-100-120 NPK $\left(\mathrm{T}_{1}\right)$ y 120-100-120 NPK $\left(\mathrm{T}_{5}\right)$. Los rendimientos de algodón rama con tratamientos orgánicos y biofertilizantes arrojaron entre $2080 \mathrm{~kg} / \mathrm{ha}\left(\mathrm{T}_{4}\right)$ y $1637,50 \mathrm{~kg} / \mathrm{ha}\left(\mathrm{T}_{2}\right)$ siendo estos rendimientos obtenidos superiores a los reportados por otros investigadores en zonas productoras de algodón de color.
\end{abstract}

Palabras clave: Algodón de color, Azotobacter sp., Azospirillum sp., biofertilizante, materia orgánica.

\begin{abstract}
The objective of the present investigation was to compare the effects of Azotobacter sp. and Azospirillum sp. in the performance of colored cotton (Gossypium barbadense L.) in Los Pichones- Tacna farm, whose effect, the experimental design of DBCA with 5 treatments and 4 Blocks was used. The treatments were: $2 \mathrm{~kg} / \mathrm{m} 2 \mathrm{M} . \mathrm{O}+60$ 100-120 NPK (T1), 2 kg / m2 M.O + Azospirillum sp. (T2), 2 kg / m2 M.O + Azotobacter sp. (T3), 2 kg / m2 M.O + Azospirillum sp. + Azotobacter sp. (T4), 120-100-120 NPK (T5). The results indicate that the yields with application of organic matter, Azospirillum sp. and Azotobacter sp (T4) are superior to the treatments $2 \mathrm{~kg} / \mathrm{m} 2 \mathrm{M} . \mathrm{O}+$ Azospirillum sp (T2) and $2 \mathrm{~kg} / \mathrm{m} 2 \mathrm{M} . \mathrm{O}+$ Azotobacter sp. (T3), these treatments being exceeded by the chemical treatments $2 \mathrm{~kg} / \mathrm{m} 2$ M.O + 60-100-120 NPK (T1) and 120-100-120 NPK (T5). The yields of branch cotton with organic treatments and biofertilizers yielded between $2080 \mathrm{~kg} / \mathrm{ha}$ (T4) and $1637,50 \mathrm{~kg} / \mathrm{ha}$ (T2), these yields being higher than those reported by other researchers in areas producing colored cotton.
\end{abstract}

Keywords: Colored cotton, Azotobacter sp., Azospirillum sp., biofertilizer, organic matter.

\footnotetext{
${ }^{1}$ Facultad de Ciencias Agropecuarias, Universidad Nacional Jorge Basadre Grohmann. Tacna-Perú. E-mail: arevalonelly@hotmail.com

${ }^{2}$ Facultad de Ciencias Agropecuarias, Universidad Nacional Jorge Basadre Groh mann. Tacna-Perú. E-mail: rosariozegarra13@hotmail.com 


\section{INTRODUCCIÓN}

El algodón es un cultivo agrícola no alimentario de mayor intercambio comercial en el ámbito mundial. En la actualidad esta planta textil se produce aproximadamente en 130 países. En los últimos 50 años, el algodón ha ocupado un $25 \%$ de la superficie agrícola del planeta, en el mundo se producen alrededor de 25 millones de toneladas de algodón al año, según lo reportado por Bautista (2006).

El algodón nativo peruano Gossypium barbadense L., denominado "Del País", es una especie cultivada en el Perú desde hace más de 2000 años a.C., por la producción de fibra de color utilizada con fines textiles y es considerada Patrimonio Genético Étnico-Cultural de la Nación (Ley N²9224).

Uno de los principales problemas que presenta el sur del país son las condiciones climáticas y edáficas, presencia de suelos salinos y sequías, dificultando de esta manera poder cultivar en dichas zonas; por estas razones, el algodón de color se presenta como una alternativa de cultivo en la costa sur del país, debido a que es un cultivo muy rústico y tiene una gran adaptación a las condiciones climáticas, a suelos salinos y presencia de sequías; así también resistencia a plagas y enfermedades. Como parte de las estrategias de una agricultura sostenible, se hace necesario disminuir el uso de fertilizantes nitrogenados de síntesis, mediante la utilización de los biofertilizantes. En particular, los géneros Azotobacter y Azospirillum son utilizados como agentes promotores de crecimiento vegetal debido a su capacidad para fijar nitrógeno atmosférico y producir hormonas de tipo indólico (Guzmán et al., 2012).

Con la aplicación de materia orgánica y de biofertilizantes (Azotobacter sp. y el Azospirillum sp.) en el algodón de color, se pretende incrementar los rendimientos. Es necesario aplicar tecnologías adecuadas buscando productividad y sostenibilidad del cultivo del algodonero en las condiciones áridas salinas de Tacna.

Actualmente, se tiene referencias de los trabajos de investigación realizados sobre biofertilización en el rendimiento de otros cultivos, donde reportan ensayos en invernadero y en campo y se demuestra el efecto positivo de la materia orgánica y biofertilizantes en el crecimiento y rendimiento de diferentes cultivos. Así, Alarcón et al. (2009) estudiaron en tomate Lycopersicon esculentum, Mill variedad "ISCAB-10" el efecto de diferentes concentraciones de la cepa comercial de Azotobacter chroococcum (INIFAT-12) comparados con un testigo sin aplicación, reportando que los mejores resultados se alcanzaron cuando se aplicó el Azotobacter chroococcum al 50\%, obteniéndose un rendimiento de 14,14 t/ha, 12,21 t/ha, con Azotobacter al 10\% y 9,39 t/ha, con el control sin aplicación. De igual manera, López et al. (2008) evaluaron el efecto de cepas nativas fijadoras de nitrógeno Azotobacter $s p$. y solubilizadora de fósforo Bacillus megatherium con adición de fertilizantes químicos en el crecimiento de maíz cv. INIA-SQ-1. Se utilizaron dos suelos de alta fertilidad (suelo A) y de baja fertilidad (suelo B). Los resultados, en el suelo A, indican que la altura de planta fue estadísticamente superior en los tratamientos $2(108,1 \mathrm{~cm})$ fertilización química y tratamiento 3 fertilización química y Azotobacter $(107,2 \mathrm{~cm})$, seguido del tratamiento 5 fertilización química sin fósforo $(101,9 \mathrm{~cm})$. En el suelo $\mathrm{B}$, también se logró la mayor altura de la planta con el tratamiento 3, seguido por los tratamientos 2 y 7 (tratamiento de fertilizante químico con adición de inóculo de fósforo).

También Constantino et al. (2010), en papaya Carica papaya variedad Maradol, evaluaron el efecto de tres biofertilizantes aplicados solos o en combinación con Azotobacter chroococcum, Azotobacter brasilense y Glomus intraradices, y un biorregulador del crecimiento vegetal, el ácido giberélico $\left(\mathrm{AG}_{3}\right)$. Los tratamientos simples con $A$. chroococcum y $A$. brasilense, incrementaron el porcentaje de germinación a 90,28 y $88,89 \%$ respectivamente. Además, con la aplicación de los biofertilizantes y el $A G_{3}$, la velocidad de germinación se incrementó y el tiempo medio de germinación se redujo. Asimismo, Padilla et al. (2006) investigaron el efecto de 3 biofertilizantes adicionados con otros compuestos y microorganismos (Z-Plex, Soil-Plex, Maya Magic) en el cultivo de melón Cucumis melo. Resaltando el Maya Magic que está conformado por microorganismos fijadores de nitrógeno como Azotobacter, Clostridium, Cianófitas, algas marinas entre otros.

Asimismo, Rivera et al. (2010) compararon en naranjo agrio Citrus aurantium la efectividad de la cáscara de naranja, la cachaza y el estiércol de pollo combinados con biofertilizantes constituidos por cepas de Azospirillum, Azotobacter y bacterias solubilizadoras de fósforo, encontrando que el crecimiento de la planta fue directamente proporcional a la dosis aplicada de biofertilizante.

Por otro lado, Rincón et al. (2004) comprobaron en el cultivo de pimiento que la utilización del estiércol, biofertilizante Azobac a dosis de 15 1/ha junto con una fertilización nitrogenada ha resultado ser eficaz, consiguiéndose la mejor producción y calidad del fruto. Borda et al. (2011) experimentaron en el cultivo de Stevia rebaudiana, aplicando materia orgánica 30 t/ha logrando un rendimiento de $1538 \mathrm{~kg} / \mathrm{ha}$ y con la aplicación de 30 t/ha de materia orgánica más Azotobacter obtuvo $700 \mathrm{~kg} / \mathrm{ha}$.

Gómez (2009) utilizó tecnología orgánica con Biofit, que es un preparado con té de composta, el cual contiene microorganismos fijadores de nitrógeno y otros microorganismos benéficos encontrando un incremento de $11,6 \%$ en comparación al tratamiento testigo. 
Asimismo, Castilla et al. (2009) realizó una investigación en caña de azúcar en soca y plantación de primer corte con el objetivo de buscar tecnologías que conduzcan a reducir el alto costo de las fertilizaciones inorgánicas y el impacto ambiental causado en estas, por lo cual evaluaron, desde el año 2002 la biofertilización como una alternativa en la nutrición vegetal.

Por otro lado, Piñero et al. (2011) realizaron un experimento en el cultivo de pimiento Capsicum annuum L., utilizando Biopron, que contiene bacterias promotoras del crecimiento Azospirillum brasilense y Pantoea encontró que con la aplicación del Biopron obtuvo un peso seco por planta de $60 \mathrm{~g}$, el cual, no afectó de manera significativa al crecimiento vegetativo respecto a las plantas no inoculadas que arrojó un peso de $50 \mathrm{~g}$.

También Araujo et al. (2010) evaluaron el efecto del biofertilizante Azotobacter sp. como alternativa de fertilización en papa. El rendimiento del cultivo de papa, usando el biofertilizante Azotobacter sp. estuvo dentro del promedio encontrado en la zona de estudio con la fertilización tradicional (25-30 $\mathrm{t} / \mathrm{ha})$, teniendo una reducción del $30 \%$ de los costos de producción del cultivo.

Del mismo modo, Rodríguez et al. (2010) evaluaron la fertilización química, orgánica y biofertilización en la nutrición y rendimiento del ají Capsicum $s p$. Los resultados mostraron que la fertilización química, así como la orgánica y la biofertilización, dieron los mejores resultados.

En la región Lambayeque, se realizó la evaluación fitosanitaria y potencial de rendimiento de algodones nativos de color marrón aplicando 600 gramos de humus, reportando un rendimiento de algodón rama $0,96 \mathrm{~kg} /$ planta, reportado por Acuña (2009).

Como es de conocimiento la tendencia mundial dentro del marco de una agricultura sostenible aboga por tecnologías que contribuyan con la recuperación y mantenimiento del suelo mediante estrategias que mejoren su calidad y productividad. La utilización de microorganismos con potencial biofertilizante es una excelente alternativa para el desarrollo de una agricultura sustentable. El uso de fertilizantes biológicos permite incorporar poblaciones benéficas y eficientes, con el fin de restaurar las propiedades de los suelos y la dinámica rizósferica deteriorada por el manejo tradicional del mismo. Por todo lo mencionado anteriormente el objetivo de la presente investigación fue comparar el efecto de Azotobacter sp. y Azospirillum sp. en el rendimiento del algodón de color en el fundo Los Pichones-Tacna - 2015.

\section{MATERIAL Y MÉTODOS}

\section{Material vegetal}

La población del experimento estuvo constituida por 180 plantas, cuyo material genético proviene de la colección de ecotipos de algodón de color Gossypium barbadense L. tipo áspero, evaluados anteriormente durante 3 campañas en el fundo los Pichones. Este material fue colectado de las zonas de Tacna ( $\mathrm{La}$ Yarada, Sama y Los Palos).

La muestra fue tomada al azar y estuvo constituida por 120 plantas de algodón de color correspondiendo a un $66,67 \%$ de la población.

Tipo y diseño de la investigación

El tipo y diseño de investigación fue experimental, ya que se emplearon las variables independientes (biofertilizante y materia orgánica) para comparar los efectos en la variable dependiente rendimiento de algodón de color.

El trabajo de investigación se realizó en el fundo Los Pichones de propiedad de la Facultad de Ciencias Agropecuarias de la Universidad Nacional Jorge Basadre Grohmann de Tacna, siendo las coordenadas geográficas: latitud Sur $17^{\circ} 59^{\prime} 38^{\prime \prime}$, longitud oeste $74^{\circ} 14^{\prime} 22$ ", altitud 550 m s.n.m.

\section{Diseño experimental}

Se empleó el diseño experimental de bloques completos al azar (DBCA). Se tuvo 5 tratamientos y 4 bloques como se observa en la tabla 1, la unidad experimental fue de $6 \mathrm{~m}^{2}$ (3,0 m de largo y 2,0 $\mathrm{m}$ de ancho) con 9 plantas por unidad experimental (U.E.)

Tabla 1. Tratamientos de Azotobacter sp. y Azospirillum sp en el rendimiento de algodón de color (Gossypium barbadense L.) enel fundo Los Pichones, Tacna 2015

\begin{tabular}{|l|c|c|c|c|}
\hline Tratamientos & $\begin{array}{c}\text { Materia } \\
\text { orgánica }\end{array}$ & Fertilización & $\begin{array}{c}\text { Azotobacter } \\
\text { (kg/ha) }\end{array}$ & $\begin{array}{c}\text { Azaspirillum } \\
\text { (kg/ha) }\end{array}$ \\
\hline$T_{1}$ & $2 \mathrm{~kg} / \mathrm{m}^{2}$ & $\begin{array}{c}60-100-120 \\
\text { NPK }\end{array}$ & 0 & 0 \\
\hline$T_{2}$ & $2 \mathrm{~kg} / \mathrm{m}^{2}$ & 0 & 0 & 1 \\
\hline$T_{3}$ & $2 \mathrm{~kg} / \mathrm{m}^{2}$ & 0 & 1 & 0 \\
\hline$T_{4}$ & $2 \mathrm{~kg} / \mathrm{m}^{2}$ & 0 & 1 & 1 \\
\hline$T_{5}$ & 0 & $\begin{array}{c}120-100-120 \\
\text { NPK }\end{array}$ & 0 & 0 \\
\hline
\end{tabular}

Fuente: Elaboración propia

\section{Manejo agronómico \\ Invernadero (producción de plántulas)}

Inoculación de las semillas. Primero se realizó el tratamiento de las semillas, remojándolas con agua caliente a $40^{\circ} \mathrm{C}$ por tres días para obtener semillas pre germinadas, estas semillas pre germinadas se colocaron en una solución de Azotobacter sp. y Azospirillum sp. según tratamiento, se embebieron por un tiempo de 30 minutos y luego se efectuó la siembra. 
Las figuras del 1 al 4 muestran el procedimiento realizado.

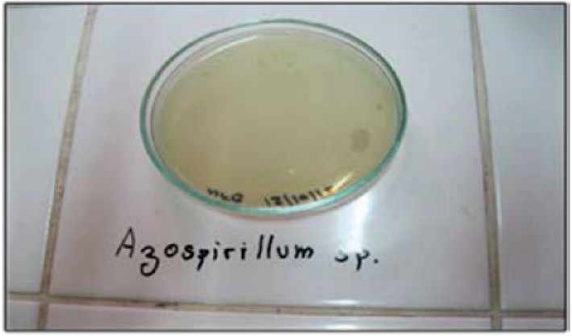

Figura 1. Inoculación con Azospirillum sp. Foto 1. Arévalo, N. (2015)

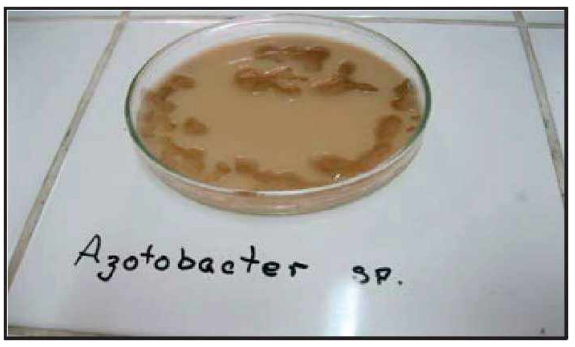

Figura 2. Inoculación con Azotobacter sp. Foto 2. Arévalo, N. (2015)

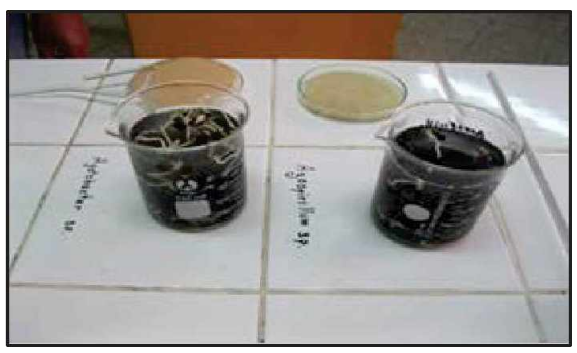

Figura 3. Inoculación con Azospirillum sp. y Azotobactersp.

Foto 3. Arévalo, N. (2015)

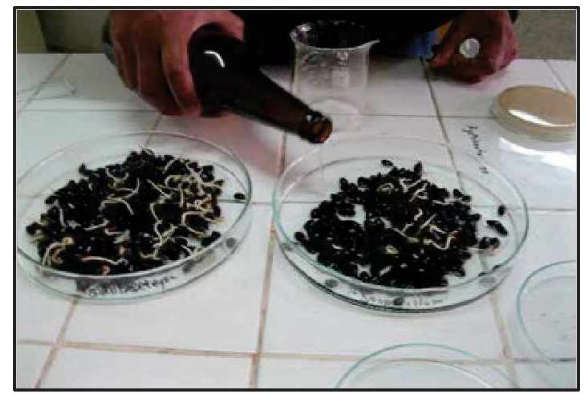

Figura 4. Inoculación de tratamientos Foto 4. Arévalo, N. (2015)

\section{Preparación del sustrato}

La preparación del sustrato consistió de una mezcla de turba con adición de compost 1:1 mezclándose con melaza como adherente. Luego se humedeció el sustrato hasta tener una capacidad de campo. En seguida, se añadió los biofertilizantes según tratamientos, en el caso de los Azotobacter sp. se cubrió con un plástico negro al sustrato preparado por un tiempo de 72 horas para permitir la fermentación del sustrato. Luego de este periodo, se retiró el plástico para orear el sustrato. Este sustrato preparado sirvió para el llenado de vasos de plástico con un volumen de $100 \mathrm{~g}$.
Siembra. La siembra de las semillas pre germinadas e inoculadas con los biofertilizantes se colocaron en los vasos que contenía el sustrato, poniéndose a una profundidad de $2 \mathrm{~cm}$, realizándose posteriormente los riegos con una frecuencia de 2 veces por semana, permaneciendo las plántulas en invernadero hasta los 35 días.

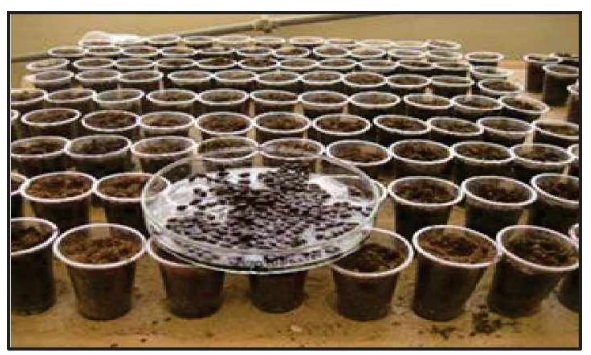

Figura 5. Siembra previa inoculación Foto 5. Arévalo, N. (2015)

Campo definitivo. Previa selección por vigor de plántulas, se llevó a campo definitivo cuando las plántulas alcanzaron un tamaño de 10 a $12 \mathrm{~cm}$.

Conducción del experimento. La selección de las plántulas para el trasplante, la preparación del suelo y las labores agronómicas se realizaron de acuerdo a las normas establecidas a las buenas prácticas agrícolas. Se retiraron los vasos de plástico y se colocaron las plántulas con todo el sustrato en hoyos $\mathrm{y}$ se trasplantaron a campo definitivo a un distanciamiento de $1,0 \mathrm{~m}$ por $1,0 \mathrm{~m}$ entre líneas y plantas respectivamente (figuras 6,7 y 8 ).

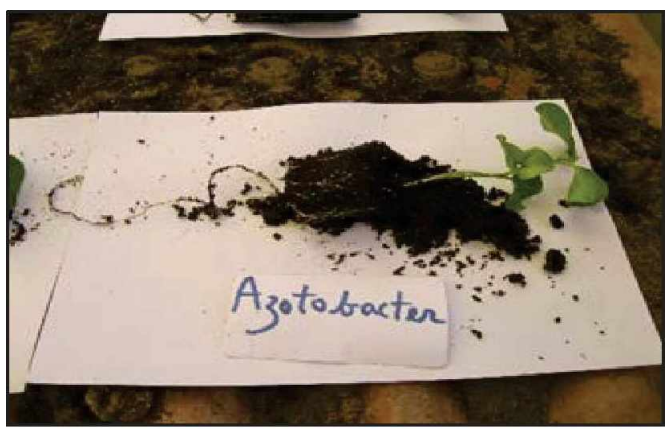

Figura 6. Plántula con Azotobacter sp.

Foto 6. Arévalo, N. (2015)

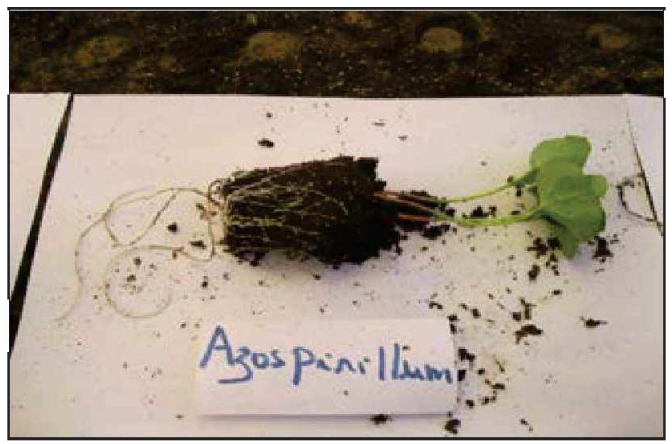

Figura 7. Plántula con Azospirilum sp. Foto 7. Arévalo, N. (2015) 


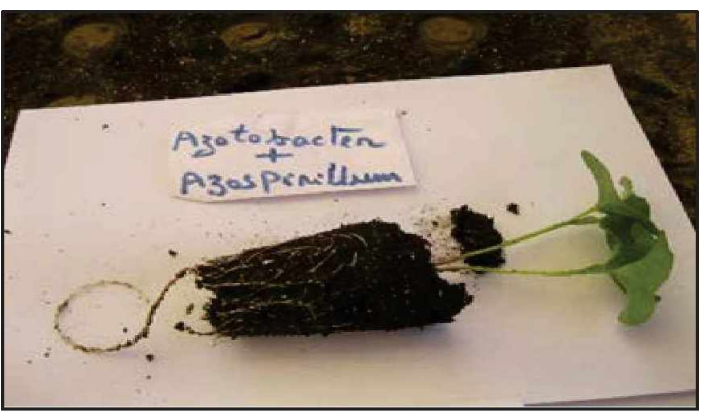

Figura 8. Plántulas con Azospirillum sp. +Azotobacter sp Foto 8. Arévalo, N. (2015)

En la etapa juvenil (5 hojas verdaderas), se aplicó la materia orgánica (M.O.) proveniente de ganado vacuno y rastrojos de cosecha, en forma de compost, a razón de $2 \mathrm{~kg} / \mathrm{m}^{2}$ a cada tratamiento.

También se aplicó Biol al $3 \%$ por cuatro veces. A los 90 días, después del trasplante, se realizó el muestreo de suelos en la zona de la rizósfera para evaluar la presencia de las bacterias inoculadas Azotobacter sp. y Azospirillum sp. utilizando los medios respectivos.

El experimento se condujo con riego por goteo, empleándose una frecuencia de 2 riegos por semana, se controlaron las plagas y enfermedades empleando productos orgánicos como: jabón potásico, biocidas y otros preparados orgánicos, con una frecuencia de 15 días.

El control de malezas se efectuó manualmente en tres oportunidades. También se realizó la poda de hojas basales (Hasta el nudo 9. ${ }^{\text {oo }}$ ).

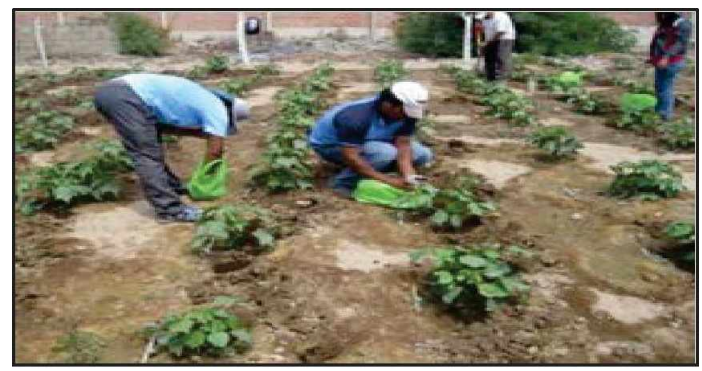

Figura 9. Aplicación de tratamientos orgánicos Foto 9. Arévalo, N. (2015)

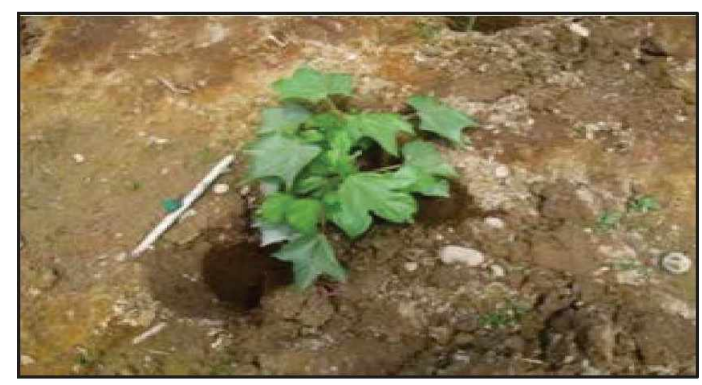

Figura 10. Planta en estado juvenil Foto 10. Arévalo, N. (2015)

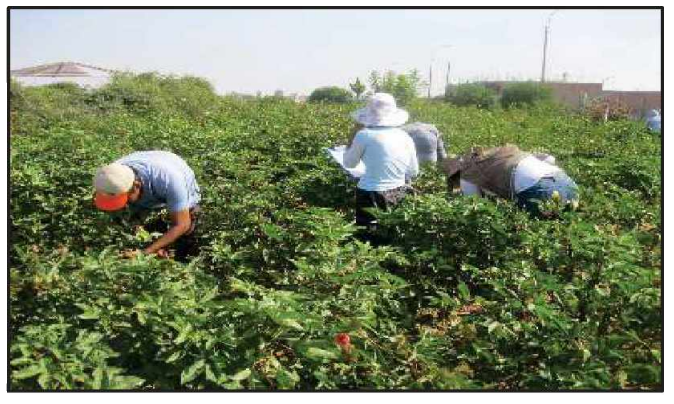

Figura 11. Planta en estado adulto

Foto 11. Arévalo, N. (2015)

\section{Características evaluadas}

La metodología aplicada para la recolección de datos fue el muestreo aleatorio simple, se marcaron 6 plantas por tratamiento y se realizaron las siguientes observaciones:

- Altura de plantas (cm): para la evaluación de esta variable se consideró medir desde el cuello de la planta hasta el brote de mayor altura, realizando esta evaluación a los 135 días.

- Número de bellotas: consistió en contar el número de bellotas por planta de una muestra de 6 plantas tomadas al azar.

- Peso de algodón rama: consistió en pesar el peso total de la cosecha tanto de fibra y semilla por planta, la cual se expresó en gramos.

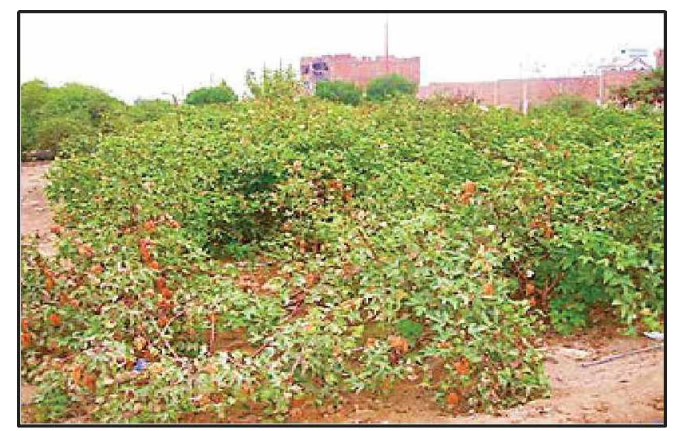

Figura 12. Planta en estado de cosecha.

Foto 12. Arévalo, N. (2015)

\section{Procesamiento $y$ análisis de datos}

Las pruebas estadísticas utilizadas fueron: ANDEVA a un nivel de significación de $5 \%,(\alpha=0,05)$. Para determinar diferencias de los tratamientos, se utilizó la prueba de Significación de Duncan a un nivel de significación $\alpha=0,05$.

Para analizar estas pruebas, se utilizó el paquete estadístico Infostat Versión Estudiantil.

\section{RESULTADOS}

\section{Altura de planta (cm)}

Los resultados del análisis de varianza con un coeficiente de variabilidad (CV) de $2,38 \%$, muestran que no se encontraron diferencias significativas para los bloques y para los tratamientos con la aplicación de materia orgánica, Azotobacter sp., Azospirillum sp. y fertilización química; a pesar de ello, se efectuó la 
Prueba de Significación de Duncan para observar la altura de las plantas con los tratamientos en estudio.

Los tratamientos $2 \mathrm{~kg} / \mathrm{m}^{2}+60-100-120$ NPK $\left(\mathrm{T}_{1}\right)$ y 2 $\mathrm{kg} / \mathrm{m}^{2} \quad+$ Azotobacter $\left(\mathrm{T}_{3}\right)$ son similares estadísticamente, siendo superiores a los tratamientos $2 \mathrm{~kg} / \mathrm{m}^{2}+$ Azospirillum $\left(\mathrm{T}_{2}\right), 2 \mathrm{~kg} / \mathrm{m}^{2}+$ Azotobacter+ Azospirillum (T 4 ) y 120-100-120 NPK ( $\mathrm{T}_{5}$ ). La mayor media se obtuvo con el tratamiento $2 \mathrm{~kg} / \mathrm{m}^{2}+60-100$ 120 NPK $\left(\mathrm{T}_{1}\right) \quad 138,45 \mathrm{~cm}$ y la media más baja con el tratamiento $120-100-120 \mathrm{NPK}\left(\mathrm{T}_{5}\right) 131,83 \mathrm{~cm}$, como se observa en la figura 13.

Tabla 2. Prueba de Significación Duncan para altura de planta $(\mathrm{cm})$ con aplicación de Azotobacter sp. y el Azospirillum sp. en el rendimiento de algodón de color (Gossypium barbadense L.) en el fundo Los Pichones, Tacna 2015

\begin{tabular}{lccc}
\hline Orden & Tratamiento & $\begin{array}{c}\text { Medias } \\
\text { (cm) }\end{array}$ & Comparación $^{1}$ \\
\hline 1 & $\mathrm{~T}_{1}$ & 138,45 & $\mathrm{~A}$ \\
2 & $\mathrm{~T}_{3}$ & 137,33 & $\mathrm{~A}$ \\
3 & $\mathrm{~T}_{2}$ & 134,93 & $\mathrm{~A} \mathrm{~B}$ \\
4 & $\mathrm{~T}_{4}$ & 134,78 & $\mathrm{~A} \mathrm{~B}$ \\
5 & $\mathrm{~T}_{5}$ & 131,83 & $\mathrm{~B}$ \\
\hline
\end{tabular}

1: Altura de planta seguidas por la misma letra no difieren por la prueba de Duncan al nivel $5 \%$ Fuente: Elaboración propia

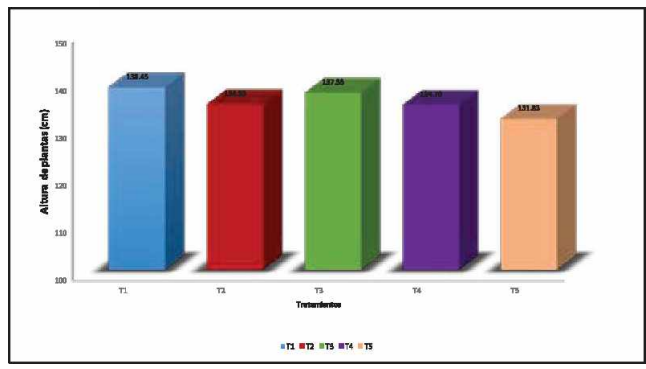

T1: 2 kg/m2 M.O + 60-100-12C NPK, T2:2 kg/m2 M.O+ Azos pirillum sp, T3:2 kg/m2 M.O + Azotobacter $s p_{\text {, }}$ T4:2 kg/m2 M.O + Azospirillum sp. +Azotobacter sp., T5:120-100-120 NPK

Figura 13. Altura de planta (cm) con aplicación de Azotobacter sp. y el Azospirillum sp. en el rendimiento de algodón de color (Gossypium barbadense L.) en el fundo Los Pichones, Tacna 2015)

Fuente: Elaboración propia

\section{Número de bellotas}

El análisis de variancia para el número de bellotas con un CV 9,08\% muestra que no se encontraron diferencias significativas para los bloques; sin embargo, se encontró diferencias significativas para los tratamientos evaluados, con aplicación de materia orgánica, Azotobacter sp., Azospirillum sp. y fertilización química, siendo necesario realizar la Prueba de Significación de Duncan para comparar los tratamientos en estudio.

Tabla 3. Prueba de Significación Duncan para número de bellotas con aplicación de Azotobacter sp. y el Azospirillum $s p$. en el rendimiento de algodón de color (Gossypium barbadense L.) en el fundo Los Pichones, Tacna 2015

\begin{tabular}{lclllll}
\hline Orden & Tratamiento & Medias & \multicolumn{2}{ll}{ Comparación $^{\mathbf{1}}$} \\
\hline 1 & $\mathrm{~T}_{5}$ & 75,75 & $\mathrm{~A}$ & & & \\
2 & $\mathrm{~T}_{1}$ & 69,25 & $\mathrm{~A}$ & & & \\
3 & $\mathrm{~T}_{4}$ & 59,00 & & $\mathrm{~B}$ & & \\
4 & $\mathrm{~T}_{3}$ & 54,75 & & & $\mathrm{~B}$ & $\mathrm{C}$ \\
5 & $\mathrm{~T}_{2}$ & 47,00 & & & & $\mathrm{C}$
\end{tabular}

$1 \longdiv { \text { Número de bellotas seguida por la misma letra no difieren por la Prueba de Duncan a } }$ nivel $5 \%$

Fuente: Elaboración propia

Los tratamientos 120-100-120 NPK $\left(\mathrm{T}_{5}\right)$ y $2 \mathrm{~kg} / \mathrm{m}^{2}$ $+60-100-120$ NPK $\left(\mathrm{T}_{1}\right)$ son similares estadísticamente, siendo superiores a los tratamientos $2 \mathrm{~kg} / \mathrm{m}^{2}+$ Azotobacter + Azospirillum $\left(\mathrm{T}_{4}\right), 2 \mathrm{~kg} / \mathrm{m}^{2}$ + Azotobacter $\left(\mathrm{T}_{3}\right)$ y $2 \mathrm{~kg} / \mathrm{m}^{2}+$ Azospirillum $\left(\mathrm{T}_{2}\right)$; $\sin$ embargo, la mayor media se obtuvo con el tratamiento 120-100-120 NPK( $\left.\mathrm{T}_{5}\right)$, con 75,75 bellotas y el más bajo el tratamiento $2 \mathrm{~kg} / \mathrm{m}^{2}+$ Azospirillum $\left(\mathrm{T}_{2}\right)$ con 47 bellotas.

\section{Peso de algodón rama}

$\mathrm{El}$ análisis de variancia para el peso de algodón rama con un CV 6,32\%, muestra que, para los bloques, no se encontraron diferencias significativas. Por otro lado, los tratamientos evaluados muestran diferencias significativas entre los tratamientos con aplicación de materia orgánica, Azotobacter sp., Azospirillum sp. y fertilización química, siendo necesario realizar la Prueba de Significación de Duncan para comparar los tratamientos en estudio.

Tabla 4. Prueba de Significación Duncan para peso de algodón rama (g) con aplicación de Azotobacter sp. y el Azospirillum sp. en el rendimiento de algodón de color (Gossypium barhadense L.) en el fundo Los Pichones, Tacna 2015

\begin{tabular}{lccc}
\hline Orden & Tratamiento & $\begin{array}{c}\text { Medias } \\
(\mathbf{g})\end{array}$ & Comparación $^{1}$ \\
\hline 1 & $\mathrm{~T}_{5}$ & 270,00 & $\mathrm{~A}$ \\
2 & $\mathrm{~T}_{1}$ & 257,50 & $\mathrm{~A}$ \\
3 & $\mathrm{~T}_{4}$ & 208,00 & $\mathrm{~B}$ \\
4 & $\mathrm{~T}_{3}$ & 191,00 & $\mathrm{~B}$ \\
5 & $\mathrm{~T}_{2}$ & 163,75 & $\mathrm{C}$ \\
\hline $\begin{array}{l}\text { 1: Pesa de algodan rama seguidas par la misma letra no difieren par la prueba de Duncan } \\
\text { al nivel 5\% }\end{array}$ \\
Fuente: Elaboración propia
\end{tabular}

El tratamiento 120-100-120 NPK $\left(\mathrm{T}_{5}\right)$, con fertilización química obtuvo el promedio más alto con $270 \mathrm{~g}$ de peso, seguido del tratamiento $2 \mathrm{~kg} / \mathrm{m}^{2}+60$ 100-120 NPK $\left(\mathrm{T}_{1}\right)$ con 257,5 $\mathrm{g}$, siendo similares estadísticamente y superiores a los tratamientos 2 $\mathrm{kg} / \mathrm{m}^{2}+$ Azotobacter+ Azospirillum $\left(\mathrm{T}_{4}\right), 2 \mathrm{~kg} / \mathrm{m}^{2}$ + Azotobacter $\left(\mathrm{T}_{3}\right)$ y $2 \mathrm{~kg} / \mathrm{m}^{2}+$ Azospirillum $\left(\mathrm{T}_{2}\right)$, con aplicación de Azotobacter sp. y Azospirillum sp. Respectivamente, el promedio más bajo lo obtuvo el tratamiento $2 \mathrm{~kg} / \mathrm{m}^{2}+$ Azospirillum $\left(\mathrm{T}_{2}\right)$ con $163,75 \mathrm{~g}$.

En la figura 14, se observa el rendimiento en $\mathrm{kg} / \mathrm{ha}$ de los tratamientos en estudio donde destaca el tratamiento $120-100-120 \mathrm{NPK}\left(\mathrm{T}_{5}\right)$. 


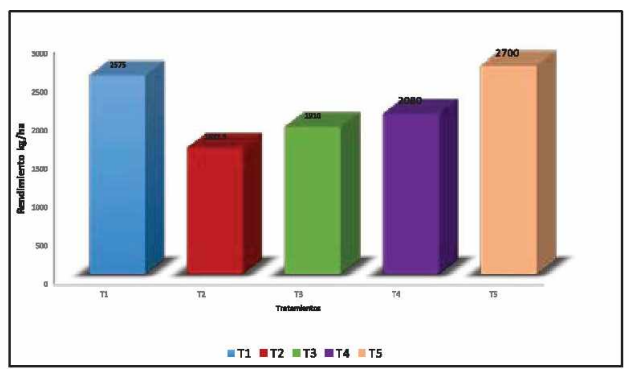

T1: 2 kg/m2 M.0 + 60-100-120 NPK, T2:2 kg/m2 M.0+ Azospirillum sp, T3:2 kg/m2 M.O + Azotobacter sp, T4:2 kg/m2 M.O + Azospirillum sp. +Azotobacter sp., T5:120-100-120 NPK

Figura 14. Rendimiento kg/ha con aplicación de Azotobacter sp. y el Azospirillum sp. en el rendimiento de algodón de color (Gossypium barbadense L.) en el fundo Los Pichones, Tacna 2015

Fuente: Elaboración propia

\section{ANÁLISIS Y DISCUSIÓN DE RESULTADOS}

\section{Altura de plantas}

En lo que respecta a la altura de plantas, no existe diferencias estadísticas entre los tratamientos orgánicos e inorgánicos. Estos resultados son similares a los encontrados por Ruiz et al. (2007), quienes empleando cinco abonos orgánicos en el cultivo de cebolla, reportan que la aplicación de estiércol de caprino a razón de 30 tha tiene influencia sobre las variables de crecimiento, aun cuando no se encontró diferencias significativas en algunos de los casos. La altura de plantas es una característica que depende del factor genético, propios del genotipo. Así también, Egas (2010) trabajó en cacao Theobroma cacao L., donde comparó cepas introducidas, cepas nativas de Azotobacter $s p$. y fertilización química, concluyendo que la fertilización química es similar a la aplicación con cepas introducidas de Azotobacter sp., logrando una altura de plantas de $35,60 \mathrm{~cm}$ y con fertilización química $34,59 \mathrm{~cm}$.

De igual manera, Adriano et al. (2011) trabajaron en café Coffea arábica L., variedad Bourbon con el uso de 3 biofertilizantes (cepas PACHAZ08 de Azotobacter sp., cepa 11B de Azospirillum y hongos formadores de micorrizas arbusculares HMA) reportan que la aplicación de los 3 biofertilizantes tuvo efecto positivo en el crecimiento y desarrollo, destacando los tratamientos $\mathrm{T}_{2}$ (Azospirillum) con $17,2 \mathrm{~cm}$ y $\mathrm{T}_{8}$ (Azospirillum con Azotobacter y HMA) con $17,8 \mathrm{~cm}$.

\section{Número de bellotas}

Con respecto al número de bellotas los tratamientos con fertilización química registraron valores superiores a los tratamientos orgánicos. Así, se resalta el tratamiento químico T5 con 75,75 bellotas y el tratamiento $\mathrm{T} 1$ con 69,25 bellotas. Estos resultados son contrarios a lo reportado por Rodríguez et al., (2010), en ají jalapeño que, combinando fertilización química, fertilización orgánica y biofertilización, obtuvieron un incremento en el número de frutos y peso por planta superior a los tratamientos con solo fertilización química.
Asimismo, Pellicer et al. (2008), trabajando en pimiento con materia orgánica líquida y bacterias fijadoras de nitrógeno (Azotobacter y Azospirillum), encontraron que no hubo diferencias significativas en la producción total de frutos, de $12,05 \mathrm{~kg} / \mathrm{m}^{2}$ en el tratamiento con biofertilizante y de $10,85 \mathrm{~kg} / \mathrm{m}^{2}$ para el tratamiento sin biofertilizante.

\section{Peso de bellota}

Los resultados indican que la aplicación combinada de materia orgánica, Azospirillum y Azotobacter sp., obtuvo 208 gramos de bellota por planta, siendo superiores a los tratamientos orgánicos $\mathrm{T}_{3}$ y $\mathrm{T}_{2} ; \sin$ embargo, fueron superados por los tratamientos químicos que registraron pesos de $270 \mathrm{~g}$ y $257,50 \mathrm{~g}\left(\mathrm{~T}_{1}\right.$ y $\mathrm{T}_{5}$ respectivamente). Estos resultados son similares a los reportados por Nicolalde y Quintana (2010) que experimentaron en el cultivo de brócoli, utilizando 2 biofertilizantes (Azotolic y Azotolic Plus) con 3 dosis y 3 fórmulas de fertilizantes químicos con 2 testigos, quienes reportan que los mayores rendimientos se obtuvieron con los niveles más altos de fertilización química pertenecientes al $100 \%$, resultando ser el mejor el $\mathrm{T}_{7}\left(180 \mathrm{Kg}\right.$ de $\mathrm{N} / \mathrm{ha}, 60 \mathrm{Kg}$ de $\left.\mathrm{P}_{2} \mathrm{O}_{5} / \mathrm{ha}\right)$, con $3 \mathrm{ml} / 1$ de Azototic logrando 21 tha.

Asimismo, los resultados que se obtuvieron en el presente trabajo reflejan que los tratamientos orgánicos e inorgánicos fueron superiores a los reportados por Acuña (2009) quien obtuvo un rendimiento de algodón rama $1279 \mathrm{~kg} / \mathrm{ha}$ tanto en campaña normal y soca, con ecotipos de algodón de color marrón aplicando humus $600 \mathrm{~g} /$ planta.

También Cáceres (2010) empleó fertilización inorgánica, orgánica y biofertilizante en cultivo de algodón cv. Guazuncho 2000, utilizando 11 tratamientos, los rendimientos de algodón rama indican que el tratamiento $\mathrm{T}_{4}$ arroja un rendimiento de $1278,5 \mathrm{~kg} / \mathrm{ha}$, el tratamiento $T_{9}$ con $1273,2 \mathrm{~kg} / \mathrm{ha}$ y el tratamiento $T_{11}$ con $1237,4 \mathrm{~kg} / \mathrm{ha}$.

Igualmente, Rincon et al. (2004) comprobaron en el cultivo de pimiento que la utilización del biofertilizante Azobac a 15 1/ha junto con una fertilización nitrogenada obtuvieron la misma producción y calidad de fruto, donde se incorporó $50 \%$ de nitrógeno mineral más $15 \mathrm{l} / \mathrm{ha}$ de biofertilizante, logrando un rendimiento de $15,6 \mathrm{~kg} / \mathrm{m}^{2}$ en comparación al tratamiento de $100 \%$ de nitrógeno mineral que tuvo un rendimiento de $15,7 \mathrm{~kg} / \mathrm{m}^{2}$.

En cambio, Araujo et al. (2010) en cultivo de papa, empleando solo el biofertilizante Azotobacter $s p$. reportan rendimientos de 25 a 30 t/ha similares al testigo con fertilización química. Además, indican una reducción del $30 \%$ en los costos de producción. Asimismo, Alarcón et al. (2009) señalan que los mejores rendimientos en tomate se alcanzaron aplicando altas concentraciones de Azotobacter contrariamente a los obtenidos en esta investigación. 
También es necesario indicar que en los tratamientos inorgánicos, debido a la aplicación del nitrógeno, las ramas fruteras tuvieron mayor desarrollo (ramas multipodiales), generando de esta manera, un mayor número de bellotas.

\section{CONCLUSIONES}

Los tratamientos con fertilización inorgánica obtuvieron los mayores rendimientos de algodón rama (número y peso de bellotas) para el presente ensayo. Los procedimientos de Azotobacter y Azospirillum en mezcla obtuvieron los mejores rendimientos dentro de los tratamientos orgánicos.

El método con Azospirillum obtuvo el menor rendimiento para el presente ensayo.

Respecto a la altura de plantas, no se encontraron diferencias estadísticas entre los tratamientos orgánicos e inorgánicos.

En cuanto al número de bellotas, los tratamientos con fertilización química obtuvieron mayores valores.

\section{RECOMENDACIONES}

Teniendo en cuenta que debemos conservar el medio ambiente con un desarrollo sostenible, se recomienda: Investigar con otras fuentes orgánicas como humus de lombriz, abonos verdes, entre otros, con dosis y momentos de aplicación para evaluar sus efectos y sostenibilidad sobre las propiedades físicas, químicas, biológicas y en el rendimiento de algodón de color. Efectuar indagaciones con fuentes orgánicas en suelos con problema de salinidad.

Producir colecciones de algodón de color en diferentes zonas del país para formar un banco de germoplasma y así preservar el recurso genético del algodón de color Fomentar como línea de investigación en la Escuela de Agronomía, empleando sistemas de cultivo como policultivo y rotacional, con el uso de productos orgánicos tendiente a una cultura orgánica.

\section{REFERENCIAS BIBLIOGRÁFICAS}

Acuña, B. (2009). Evaluación fitosanitaria y potencial de rendimiento de algodones nativos de color en Lambayeque-Peru. (tesis de maestría) Universidad Federal de Pelotas. Rio Grande do Sul, Brasil.

Adriano, M., Jarquín, R. Hernandez C., Salvador M., Monreal C. (2011). Biofertilización de café orgánico en etapa de vivero en Chiapas, México. Revista Mexicana de Ciencias Agricolas, 2(3), 417-431.

Alarcón A., Godefoy M. y González G. (2009). Efecto de diferentes concentraciones de Azotobacter chroococcum sobre algunos parámetros del crecimiento y el rendimiento del tomate (Lycopersicon esculentum, Mill). Revista Electrónica Granma Ciencia, 13(1).

Alarcón, A. y Ferrera, R. (2000). Biofertilizantes: Importancia y utilización en la agricultura.
Agricultura Técnica en México, 26(2), 191203.

Araujo, Y., Diaz, L., Rodriguez, F., Pargas, L. (2010). Efecto del biofertilizante Azotobacter sp. en el cultivo de papa en el estado Mérida. Instituto Nacional de Investigaciones Agrícolas del Estado Mérida. Av. Urdaneta, Mérida, Venezuela.

Bautista, M. (2006). Estudio de rentabilidad del cultivo algodonero (Gossypium hirsutum L.) utilizando la variedad transgénica $448 \mathrm{~B}$, en el tejido Luchana, municipio de San Pedro, Coahuila. (tesis de pregrado) Universidad Autónoma Agraria Antonio Navarro, Torreón Coahuila. México.

Borda, D., Pardo, J. Martinez, M., Montaña, J. (2011). Producción de un biofertilizante a partir de un aislamiento de Azotobacter nigricans obtenido en un cultivo de Stevia rebaudiana Bert. Universitas Scientianum, 14(1).

Cáceres, R. (2010). Respuesta a la fertilización orgánica e inorgánica del algodón en el Sur Oeste del Chaco. Las Breñas, Argentina: EEA INTA.

Castilla, L., Moller A., Barona, G. y Hernandez, L. (2009). Avances en la respuesta del cultivo de caña de azúcar, a la biofertilización con bacterias fijadoras de nitrógeno y hongo solubilizador de fosforo, Riopaila-Castilla S.A. Valle del Cauca, Colombia. Ponencia presentada al VIII Congreso de la Asociación Colombiana de Técnicos de la Caña de Azúcar. Colombia.

Egas, J. (2010). Efecto de la inoculación con Azotobacter sp. en el crecimiento de plantas injertadas de cacao (Theobroma cacao) Genotipo nacional, en la provincia de Esmeraldas. (tesis de pregrado). Escuela Politécnica Nacional. Quito.

Gómez, M. (2009). Efecto de una tecnología orgánica Biofit sobre la producción y calidad de un cultivar de rosa variedad Freedom. (tesis de pregrado). Universidad Pontificia Javeriana. Facultad de Ciencias, Bogotá.

Guzmán, A., Obando, M., Rivera, D., y Bonilla, R. (2012). Selección y caracterización de rizobacterias promotoras de crecimiento vegetal (RPCV) asociadas al cultivo de algodón (Gossypium hirsutum). Revista colombiana de Biotecnología, 16, 182-190.

Lopez, M., Martínez, R., Brossard, M., Bolívar, A., Alfonso, N., Alba, A. Pereira, H. (2008). Efecto de biofertilizantes bacterianos sobre el crecimiento de un cultivar de maíz en dos suelos contrastantes venezolanos. Agronomía Tropical, $58(4)$.

Nicolalde, A. y Quintana, D. (2010). Utilización de hacterias fijadoras de nitrógeno (Azotobacter) y solubilizadoras de fósforo en el cultivo de brócoli (Brassica oleracea) var. Legacy en Otavalo. (tesis de pregrado). Universidad Técnica del Norte. Ecuador.

Padilla, E., Esqueda, M., Sánchez, A., Troncoso, R. y Sánchez, A. (2006). Efecto de biofertilizantes en 
el cultivo de melón con acolchado plástico. Revista Fitotecnia Mexicana. 29(4), 321-329.

Pellicer, C., Perez, A., Abadia, A., Rincon, L., Paredes, A., Carrillo, F. (2008). Resultado del aporte de biofertilizantes a un cultivo de pimiento con fertilización ecológica. Ponencia presentada VIII Congreso SEAE, IV Congreso Iberoamericano Agroecología. Bullas-Murcia.

Piñero, C., Cuadra, P., Marín, M., Amor, F., (2011). Inoculación con bacterias promotoras del crecimiento en la producción y calidad del pimiento. Ponencia presentada a X Reunión de la Red Buena. Albacete.

Rincon, A., Perez, A., Abadia, C., Pellicer, A. y Valero, A. (2004). El uso de biofertilizantes en la fertilización nitrogenada de los cultivos hortícolas. Agricultura. 879,788-792.

Rivera, M., Trujillo, A., y Pereyra, A. (2010). Los biofertilizantes integrados con bacterias fijadoras de $\mathrm{N}$, solubilizadoras de $\mathrm{P}$ y sustratos orgánicos en el crecimiento de naranjo agrio Citrus aurantium. Inverciencia, 35(02).

Rodríguez, E., Bolaños, M., Menjivar, J. (2010). Efecto de la fertilización en la nutrición y rendimiento de aji (Capsicum sp.) en el Valle del Cauca, Colombia Esp. Cipag 10. Acta agronómica, 59(1), 55-6.

Ruíz, C., Russián, T. y Tua, D. (2007). Efecto de la fertilización orgánica en el cultivo de la cebolla. Agronomia Tropical, 57(1), 7-14. 